\title{
Maintaining Quality of Candidate Strains of Transgenic Mosquitoes for Studies in Containment Facilities in Disease Endemic Countries
}

\author{
John D. Mumford, ${ }^{1}$ Adrian W. Leach,, Mark Q. Benedict, ${ }^{2}$ Luca Facchinelli, and M. Megan Quinlan ${ }^{1}$
}

\begin{abstract}
Transgenic mosquitoes are being developed as novel components of area-wide approaches to vector-borne disease control. Best practice is to develop these in phases, beginning with laboratory studies, before moving to field testing and inclusion in control programs, to ensure safety and prevent costly field testing of unsuitable strains. The process of identifying and developing good candidate strains requires maintenance of transgenic colonies over many generations in containment facilities. By working in disease endemic countries with target vector populations, laboratory strains may be developed and selected for properties that will enhance intended control efficacy in the next phase, while avoiding traits that introduce unnecessary risks. Candidate strains aiming toward field use must consistently achieve established performance criteria, throughout the process of scaling up from small study colonies to production of sufficient numbers for field testing and possible open release. Maintenance of a consistent quality can be demonstrated by a set of insect quality and insectary operating indicators, measured over time at predetermined intervals. These indicators: inform comparability of studies using various candidate strains at different times and locations; provide evidence of conformity relevant to compliance with terms of approval for regulated use; and can be used to validate some assumptions related to risk assessments covering the contained phase and for release into the environment.
\end{abstract}

Keywords: containment, insectary, mosquitoes, quality assurance, transgenic

\section{Introduction}

$\mathbf{T}$ RANSGENIC MOSQUiTOES are being developed as part of a wide-ranging set of novel approaches to vector-borne disease control (WHO/TDR and FNIH 2014). Preparing a strain of transgenic mosquitoes for eventual use in field control involves a long period of development, study, and maintenance in containment facilities, often including several locations. The concept of colony utility (Quinlan et al. 2018) recognizes the importance of ensuring that studies across time and space are comparable and based on key quality indicators for strains. Scientific studies carried out over time and across different laboratories must be interpreted based on maintenance of consistent outcomes measured in the colonies. At the same time, candidate strains must be compatible with the target vector populations in disease endemic countries (DEC), which display natural genetic diversity across the geographic range of the species. To facilitate this, a transgene from the laboratoryengineered colony will be introgressed into colonies established from the local target populations. At this time, transgenic strains are likely to be developed outside sub-Saharan Africa or at hub laboratories and, subsequently, transferred for further development and selection under containment in laboratories of DEC research partners. Introgression is the process of increasing the genetic similarity of the genetic background of individuals carrying a transgene to that of a target population by repeatedly crossing transgenic individuals to target population individuals. The introgressed colony bearing the desired trait will be assessed in small cage studies within containment facilities and, where appropriate, production scaled up to numbers needed for small field studies. Colonies are tested in containment to demonstrate that they do not pose hazards, such as increased insecticide resistance or other adverse properties before any regulated release.

\footnotetext{
${ }^{1}$ Centre for Environmental Policy, Imperial College London, Ascot, United Kingdom.

${ }^{2}$ Entomology Branch, Centers for Disease Control and Prevention (CDC), Atlanta, Georgia.

${ }^{3}$ Department of Vector Biology, Liverpool School of Tropical Medicine, Liverpool, United Kingdom.

(c) John D. Mumford et al. 2018 Published by Mary Ann Liebert, Inc. This is an Open Access article distributed under the terms of the Creative Commons Attribution License, which permits unrestricted use, distribution, and reproduction in any medium, provided the original work is properly cited.
} 
The candidate strains must maintain the traits that are necessary to meet their ultimate purpose as part of field control programs, while not necessarily retaining the same characteristics as the "natural" strain (Benedict et al. 2009). Introducing novel genetic traits, introgression, and laboratory rearing conditions are likely to impose selection pressures on the colonies over the many generations in contained use. This may affect the performance of the strain as a good candidate for field use, colony utility, and adherence to the regulated description and condition of the colony.

\section{Moving from Containment Facilities to Subsequent Studies}

The need for containment studies for transgenic mosquitoes is broadly recognized (WHO/TDR and FNIH 2014), whereas small-scale studies in containment face limitations in the data they provide for decision making. Decisions and actions taken during containment must anticipate the next steps toward open-field release and will affect the success of field studies and activities. Figure 1 shows the recommended phased approach to development, which is to satisfactorily answer study questions at each step against previously considered thresholds or criteria, before moving on to the next phase (Quinlan, thesis in preparation). Throughout the phases of development, transgenic mosquito colonies are, by regulation, maintained in containment as a precaution due to uncertainty around performance rather than due to any identified hazard. Completion of initial studies in a DEC containment laboratory does not mean an end to activities there. The supply of mosquitoes for studies in confined and small pilot field studies will necessarily come from the containment facility, because in most instances the transgenic strain will continue to be regulated as a research organism until a commercial exemption or an open-field permit is granted. Therefore, proper selection and maintenance of candidate strains in containment are the foundation for delivering a novel component to vector control in the field.

\section{Identifying critical performance characteristics}

Selecting a strain of transgenic mosquitoes for more extensive testing requires a decision on which criteria best reflect safety and performance in the field. Deciding which characteristics that best predict the success of the population for a specific purpose allows a series of tests to be designed that will facilitate the selection of potentially effective strains. In the context of the release of transgenic insects for control purposes, the focus is often on the influences of the transformation event itself on the phenotype, although performance characteristics are also influenced by the background genetics of the host strain and culture and release methods. Further discussion of this subject in the context of other insects is provided in an overview by Scolari et al. (2011), but here the emphasis is on mosquitoes.

It cannot be assumed that the laboratory host strain used for the initial transformation event in transgenic mosquitoes is a suitable wild-type "comparator" with which to predict and assess performance in the field. A comparator is a strain with which the transgenic strain is compared, preferably one that differs only by the absence of the transgene. It is believed that differences between the transgenic individuals and the comparator are due only to the effect of the transgene itself and best reflect phenotypic changes. However, laboratory host strains of mosquitoes have been selected, often over hundreds of generations, for various unique behaviors suited to laboratory culture. The initial selection of individuals used to found a colony will have substantially narrowed the potential genetic variability within subsequent generations. These strains are an obvious comparator that most closely resembles the transgenic mosquito, whereas the comparator itself is such a domesticated, laboratory-selected population that it may not exhibit the anticipated behaviors of wild mosquitoes. Field releases are likely to be most effective if transgenic strains are introgressed with local target populations, to ensure mating compatibility and adaptation to the natural environment. Introgression of the transgene with populations most closely resembling the target

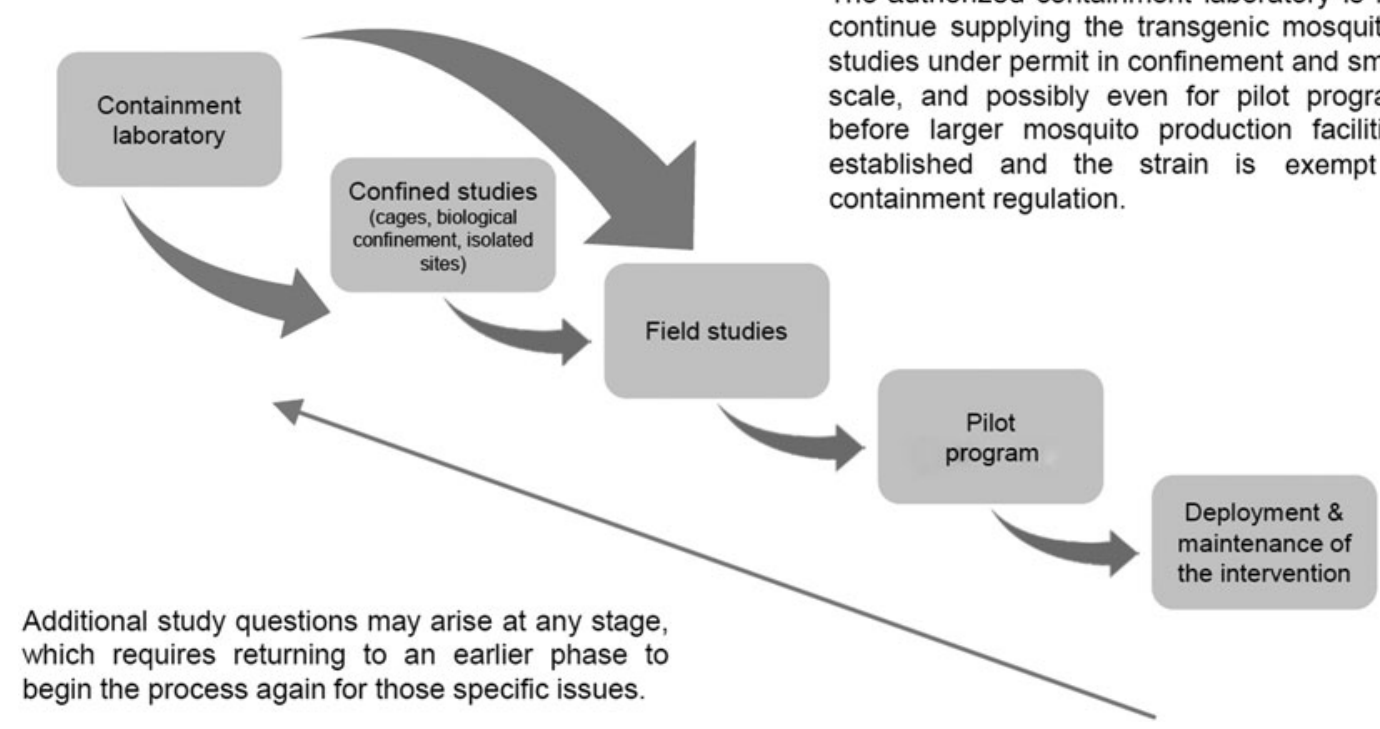

FIG. 1. The role of the containment laboratory in a phased approach to novel components of malaria vector control (Quinlan 2018). 
Table 1. Definition and Context of Key Descriptors

\begin{tabular}{lll}
\hline Descriptor & \multicolumn{1}{c}{ Definition } & Context \\
\hline Fitness & $\begin{array}{c}\text { Relative ability of individuals (or populations) to } \\
\text { survive, reproduce, and propagate genes in an } \\
\text { environment }\end{array}$ & Evolutionary selection in wild populations \\
Quality & $\begin{array}{c}\text { A standard of desired biological attributes that can be } \\
\text { monitored and controlled during maintenance of } \\
\text { insects within a contained colony }\end{array}$ & Insectary production \\
Performance & $\begin{array}{c}\text { An outcome of desired field effectiveness with respect } \\
\text { to specific management objectives }\end{array}$ & Containment studies through to field deployment \\
& P &
\end{tabular}

population is expected to occur early in the containment stage, and to be well established before field testing occurs.

Further, laboratory and production facility culture methods may detectably influence the performance of a laboratory colony. Assortative mating between types of mosquitoes has been observed to be influenced by the culture method (Paton et al. 2013) and colonization process (Reisen et al. 1985). The causes are unknown, but these observations reflect the necessity of striving for relatively natural conditions, even when one cannot identify the underlying causes that might alter performance. Feeding quality for larvae and adults and density of the rearing trays and cages can affect size, development time, competitive ability, and survival. Laboratory cultures are often produced in synchronized batches, which may select for development at a particular rate. This, along with fixed temperature and humidity regimes and constant food, may limit genetic diversity in the long term.

The terminology used to describe colony utility has been confused by conflating a term that describes the results of natural selection on genotype frequencies with direct measures of vigor such as longevity and development rate. Most notably, one term that is often used to describe colony utility is "fitness." Three evolutionary definitions are relevant in the context of maintaining quality of a colony: (1) "A biological condition in which a competing variant is increasing in frequency relative to other competing variants in a population"; (2) "A relative measure of reproductive success of an organism in passing its genes to the next generation"; and (3) "The relative ability of an individual (or population) to survive, reproduce and propagate genes in an environment." These are evolutionary concepts that predict the fate of genotypes, including any transgenes, in natural populations. Table 1 gives a definition and context for fitness, quality, and performance relevant to candidate strains in containment.

Another common use of the term "fitness" means individual vigor, and this characteristic is usually measured by life-table values such as longevity, development rate, and fecundity, but it is important when considering colony quality to keep these distinctions in mind: In the evolutionary sense of the definition, even very "unfit" colonies can have good performance relative to the intended purpose (e.g., the low standard for competitiveness needed for sterile medflies; FAO/IAEA/USDA 2003). It has been repeatedly stated that the fitness of sexually sterile males is zero, yet they are effective when used in large enough numbers for suppressing

\footnotetext{
${ }^{1}$ www.biology-online.org/dictionary/Fitness
}

target populations (e.g., with radiation-induced sterility in tephritid fruit flies). Regulators of transgenic insects may also consider high levels of fitness in a transgenic strain compared with wild populations to be a concern (European Food Safety Authority 2013). Determining the appropriate tests of fitness for a transgenic colony being considered for release requires careful distinction of the performance requirements in the field to ensure that appropriate tests are carried out. Perhaps surprisingly, the evolutionary genotypic fitness of the complete insect genotype is not an ultimate determinant of field performance for a population (European Food Safety Authority 2013).

Three general classes of genetic control approaches are currently being considered, each of which dictates appropriate performance indicators. These are described in the order of the expected degree of establishment and persistence of the transgene in the environment.

The control techniques that are the least demanding of genotypic fitness are those that have a single direct effect on the insects with which the transgenic insects interact, such as the sterile insect technique (Alphey et al. 2010) and certain variations of Release of Insects Carrying a Dominant Lethal $\left(\right.$ RIDL $\left.^{\circledR}\right)$ approach (Black et al. 2011). In these cases, persistence of the transgene in the environment is not expected; rather, the ability of a transgenic male to find females and mate, usually within a few days, is the only behavior necessary for field effectiveness. This is usually measured by mating competition studies. Longevity, fecundity, and development rate may correlate with overall strain vigor, but these characteristics are secondary to the trait that most affects performance, mating competitiveness. Fortunately, mating competitiveness can be estimated in laboratory cages (e.g., Fried 1971), although scaling up to large cages and field release can uncover deficiencies in mating behavior that are not evident at smaller scales (Davidson et al. 1970, Facchinelli et al. 2013). For the main malaria mosquito, Anopheles gambiae, evidence demonstrates that strains that perform poorly in small cages perform worse in larger ones, thus making rapid screening to eliminate unpromising strains tractable for that mosquito (Facchinelli et al. 2015).

More demanding are "self-limiting traits," in which transgenes are introduced into populations by mating, but the transgene perpetuates its effect through inheritance before disappearing at a rate determined by its overall comparative fitness. This requires not only mating competitiveness but also relatively mild loss of fitness beyond that caused intentionally by the transgene itself, if longer persistence is desired. Two such proposals have been made and modeled 
(Okamoto et al. 2014, Powell and Tabachnick 2014), and other methods (Isaacs et al. 2012, Galizi et al. 2014) demonstrate the potential to reduce pathogen development or bias sex ratio. Common approaches to discriminate fitness characteristics are life-table studies (e.g., Irvin et al. 2004) and cage studies in which the persistence of a transgene is determined in a mixed population (Harvey-Samuel et al. 2014, Valerio et al. 2016). The decline in the frequency of the transgene reflects unidentified influences that can be overlooked in discrete generation and life-table studies. Cage studies involving overlapping generations are more realistic by allowing for the effects of lifespan on mating frequency and changes in fecundity as a function of age.

The most demanding applications are those that require transgenic insects to attain the highest holistic evolutionary fitness, those in which population replacement will be accomplished without intermingling host and wild genomes nor invasive incompatibility factors. To date, no transgenic methods to accomplish this have been proposed, which highlights the fact that those developing transgenic mosquitoes do not anticipate making "a better insect" in the sense that they do not expect them to have evolutionary fitness greater than the wild type. Instead, this can be accomplished by employing gene drive (Burt 2014).

Gene drive refers to the spread of classes of genes and transgenes that can increase in frequency by favored inheritance, in spite of reduced fitness or vigor, by selectively replacing genetic sequences with the transgene at rates higher than predicted by normal processes of Mendelian inheritance (Burt 2014). Drive and segregation of the transgene from the host strain may overcome vigor deficiencies related to the host genotype, and measuring these in a nearly wild-type genetic background is essential. For these studies, the most realistic genetic background, culture methods, and testing in mesocosm environments are necessary to ensure that a realistic test has been performed. For these technologies, measures of dramatic decreases in mating competitiveness, longevity, and fecundity may be useful to eliminate "weak" strains before mesocosm studies, but a driving transgene can spread regardless of, for example, reduced competitiveness, development rate, and longevity. The overall prospects for field performance of these transgenic insects must include models to integrate the phenotypic effects and rate of spread.

In all cases, the transgenic mosquitoes must be produced at an appropriate scale, for contained use studies, and later at a greater scale for regulated field releases. This, in itself, imposes constraints that might result in compromises between performance and production, characteristics that are most evident when there is high mortality or low fecundity associated with a strain (e.g., Yamada et al. 2014). Notable deficiencies in some performance characteristics (relatively low mating competitiveness, for example) have been accepted for sterile male medflies produced around the world, ${ }^{2}$ in which programs have been successful because efficient production of very large numbers of insects has been possible. It may be less practical to make up for performance with large numbers in mosquitoes, such as

\footnotetext{
${ }^{2}$ www-naweb.iaea.org/nafa/ipc/public/tephritid-ver5-appendices .pdf
}

Anopheles, that are presently much more difficult to rear and release than fruit flies.

\section{Systems to increase the scale of laboratory tests}

As described earlier, small laboratory cages provide preliminary, but not complete, results with which to select strains for release. Standard insectaries typically provide suitable environmental conditions of temperature, humidity, and daynight cycles to entrain behaviors, including hatching, pupation, oviposition, and mating. These facilities are usually employed to maintain colonies and perform experiments in small cages, and they are appropriate for an initial screening of insect lines that are aimed at confirming the desired phenotype and discarding obvious poor performers. Ideally, this represents the first step of the progressive evaluation of a transgenic mosquito line from the laboratory where it is produced before consideration for open field release.

The lines fulfilling the performance indicators for small cage studies must go through an assessment for larger scale testing while still in containment. This is represented by increasing the scale of the evaluation process, and it is usually carried out in a dedicated facility where transgenic insects can be challenged in environmental conditions closer to those present in the open field. The InfraVEC project (Crisanti 2013) proposed facilities in Italy that would allow for the testing of transgenic mosquito strains in what was termed a confined release, which comprised large cages in environmental chambers.

Space is a key parameter that must be taken into account when scaling up the evaluation process of a transgenic insect strain. In fact, in typical small laboratory cages $(e . g ., 30 \mathrm{~cm}$ cubes), experiments are carried out in unnaturally high densities and mosquitoes are not allowed to perform some of their natural behaviors due to lack of room. For example, the large group of anopheline mosquito species, including the major malaria vectors belonging to the An. gambiae complex, mate in swarms, a behavior that cannot occur if adult mosquitoes are kept in small cages (Facchinelli et al. 2015). Although anophelines are able to mate in such a small space, this is a result of the colonization process where environmental pressure has acted on mating behavior to select stenogamy (in which mating takes place in restricted spaces such as small cages) rather than eurygamy (in which mating occurs in large spaces; Reisen et al. 1985, Charlwood 2003). Anopheline mating behavior in such conditions is different from what predominates in the field, and the results from studies aimed at revealing factors affecting mating behavior might be a poor proxy for natural behavior if adults are kept in small cages and cannot mate in swarms. Cages larger than one cubic meter (Charlwood and Jones 1980) and that also provide stimuli can reliably facilitate swarming (Marchand 1985). The use of recently colonized mosquito lines established from target field populations ensures that strains more closely related to natural field populations are used for studies related to competitive and assortative mating, which are key assays in the evaluation of lines being considered for field release.

Large cages must provide adult mosquitoes with the factors necessary to maintain them, including sources of bloodmeals, sugar feeders, resting, and oviposition sites. Increasing the environmental heterogeneity and allowing the completion of 
Table 2. Eleven Insect Quality and Insectary Operating Indicators for Contained Transgenic Mosquito Colonies

\begin{tabular}{|c|c|c|}
\hline & & Metrics/Implications \\
\hline \multicolumn{3}{|l|}{ Insect quality indicators } \\
\hline \multirow[t]{6}{*}{ Life table parameter } & Egg count & $\begin{array}{l}\text { Eggs produced per female pupa (sexed and counted in } \\
\text { previous generation); a key driver of productivity of } \\
\text { the colony }\end{array}$ \\
\hline & Egg hatch proportion & Egg survival to larvae; a first sign of quality problems \\
\hline & $\begin{array}{l}\text { Transgenic proportion in the larval } \\
\text { screen }\end{array}$ & $\begin{array}{l}\text { Comparison with expected genetic ratio; measures } \\
\text { genetic stability or drift }\end{array}$ \\
\hline & $\begin{array}{l}\text { Development duration from egg } \\
\text { hatch to pupation }\end{array}$ & $\begin{array}{l}\text { Aquatic stage development in days; main predictor of } \\
\text { batch production rate }\end{array}$ \\
\hline & Pupal sex and trait ratio & $\begin{array}{l}\text { Ratio of sexes and trait expression in newly emerged } \\
\text { pupae; gives signs of sex-linked biases and differential } \\
\text { mortality from pathogens }\end{array}$ \\
\hline & Pupal eclosion rate & $\begin{array}{l}\text { Proportion of pupae emerging after } 3 \text { days; may indicate } \\
\text { infection or contamination }\end{array}$ \\
\hline Physical condition & Wing length & $\begin{array}{l}\text { Distance between main alula and wing vein tip; an index } \\
\text { of body size that may reflect physical mating } \\
\text { compatibility and generally reflects nutrition }\end{array}$ \\
\hline Selection pressure & Insecticide resistance & $\begin{array}{l}\text { Mortality proportion against a standard dose; reflects } \\
\text { selection pressures }\end{array}$ \\
\hline \multicolumn{3}{|c|}{ Insectary operating indicators } \\
\hline \multirow[t]{3}{*}{ Insectary environment } & Temperature & Confirmation of rearing or study protocol \\
\hline & Humidity & Confirmation of rearing or study protocol \\
\hline & Lighting & Confirmation of rearing or study protocol \\
\hline
\end{tabular}

the life cycle totally within the mesocosm may increase realism of the trials conducted in them, and depending on the size, design, and purpose, may provide opportunities to use measures of behavior as performance indicators.

Outdoor cage tests may be used in some cases as a step in the evaluation of a transgenic mosquito line before limited field release (WHO/TDR and FNIH 2014). Field cages are built in areas where the target vector species is present and possibly where disease transmission occurs. Field cages provide a contained environment where the performance of a transgenic mosquito line can be challenged in natural conditions of sunlight, wind, and fluctuating temperature and humidity. These parameters can play a role in the phenotypic expression of transgenic mosquito lines showing a negative pleiotropic effect of the gene insertion that could affect the line's performance and, ultimately, the decision to move that line to an open field test (Facchinelli et al. 2013).

When moving a transgenic mosquito line to field cages and later to an open field test, the impact of strain adaptation to laboratory conditions must be carefully taken into account (Oliva et al. 2011, Paton et al. 2013). Transgene introgression in a newly colonized line with the local genetic background and its maintenance in large field cages are particularly useful to diminish laboratory adaptation and to increase the competitiveness with the wild-type comparator/target population.

\section{Quality Indicators}

Although many of the natural behaviors that are desired for mosquitoes are difficult to routinely measure in containment, it is possible to control the conditions so that several research objectives can be met. Such quality indicators cover physical and developmental metrics for the insects and the operating conditions of the insectary. Seven purposes are envisaged for such indicators:

- Experimental utility

- Maintenance of consistent quality over time

- Benchmarking between laboratories

- Benchmarking between strains

- Conformity, demonstrating compliance with potential regulatory requirements

- Validity of risk assumptions

- Management logistics

The following sections provide a list of eight useful insect quality indicators and three insectary operating indicators (Table 2), which may be monitored on a routine basis. These eight insect quality indicators are specific to the contained use of sterile male strains that contain an autosomal transgene that is maintained in populations by selecting and backcrossing transgenic females to wild-type males each generation, but they offer a general guide for indicators and insectary management capacity needed for other transgenic strains. Specific regulatory requirements on quality indicators could require extensions to the list, in particular cases.

For each indicator there is a description, a rationale, a protocol for collecting relevant data or calculating the indicator, and, where appropriate, management responses. Insectary indicators may be supported by a database with graphical summaries of data, or "database dashboards," that display tabular and graphical information to help laboratory, risk, and project managers to document, predict, or respond to the numbers and conditions of insects in the laboratories. The database can also be used to generate records that are useful in forensic examination of factors that may have affected numbers or quality in the colonies. It can also be used to generate relationships and 
values that may eventually be useful in planning a larger scale of insectary production for release purposes.

Colonies in the DEC laboratories are based on local wild types, and baselines are established once measurements for these indicators are started. Acceptable variability will need to be determined after sufficient data have been collected on each colony. The long-term trends in indicators should provide evidence on the degree of variability in physical and developmental properties of the colonies. Where trigger values for responses cannot be specified, trends in values that show changes may indicate the need for discussion and possible action on specific management responses. If phenotypic or developmental values change, it may prompt a molecular check on the genotype to compare against preliminary genotypic data.

The indicators are listed in life-stage order for mosquito biology, followed by indicators related to laboratory handling and physical conditions. The indicators for mosquito biology cover the full range of life stages to ensure that quality characteristics relevant at the different stages are included. Indicators include point values, such as egg hatch rate or pupal sex ratio, as well as broader integrating indicators, such as egg to pupa development duration. This will give a robust description of quality for colony maintenance. For other purposes, such as compliance, a smaller set of indicators may be sufficient to give a representative description of conformity of performance, for example, egg count and insecticide resistance.

\section{Egg count}

The egg count is the average number of eggs produced from adults developing from a counted batch of pupa screened and selected as female from the previous generation. It is a compound measure of quality based on egg productivity in cages that integrates female size, survival, mating success, adult feeding efficiency, and fecundity. Egg counts can be carried out by scanning digital photographs of eggs collected from an oviposition dish in each cage, or estimated by volume or weight. The number of pupae that formed the population to initiate the cage is recorded. The egg count is, therefore, a production indicator per female pupa in the parental generation. A decreasing trend in fecundity over time may mean that growing conditions in the colony are not suitable. An increase in fecundity may indicate selection pressures in the colonies that make groups of mosquitoes inconsistent over time for laboratory studies. Changes could also indicate accidental contamination of the colony or seasonal factors.

\section{Egg hatch proportion}

The egg hatch proportion is the proportion of eggs in a sample from a cohort that hatches. This is one of the several survival indicators measured at different life stages to show any change specific to the particular stages over time in colonies. In each cohort of a strain, a sample of eggs is removed and the hatching proportion is observed and recorded. The larvae resulting are kept to observe the development rate to pupation. Rapid decline in egg survival requires immediate determination of the cause, for example infection, so that the problem can be resolved. Longer trends of decreasing survival indicate poor growth conditions in the colonies and possible effects of inbreeding. Trends of increasing survival could indicate selection pressures that may lead to inconsistent results in laboratory studies over time.

\section{Transgenic proportion in the larval screen}

When colonies consist of mixtures of both transgenic and nontransgenic types, for example as a result of backcrossing, the larval screen distinguishes transgenic larvae (by fluorescent marker, for example) from nontransgenic larvae (or possibly those not exhibiting sufficient fluorescence for detection) as a proportion. This proportion is a measure of the expected genetic ratio among the progeny. The larval screen indicates whether the proportion of larvae exhibiting the transgenic trait matches the expected value (e.g., 50\% in simple inheritance). Substantial changes in this ratio could mean that there is a mistake in the screening process, there is some strong selection pressure within a backcrossed generation, or there is a loss or failure of the insertion. Late instar larvae are collected from trays and observed under a microscope/stereoscope with fluorescent lighting to detect fluorescence. The larvae are then separated into two containers and put back into separate new larval trays to continue development. If the ratio of those expressing fluorescence is substantially different from $50 \%$ (or a predefined proportion relevant to the trait), there may be mistakes in the screening process. If the proportion is too low, it may be due to a worn-out or otherwise ineffective fluorescent light source of filters in the screening process, so the light source may need to be replaced. If the proportion is too high, it may be due to misidentification of fluorescent food in the gut, which should be distinguished from the transgenic fluorescent trait. If the proportion remains out of balance after checking and correcting the screening process, then samples should be processed to confirm transgene integrity. If loss of transgene integrity is determined, then a decision would be needed on whether the line should be continued.

\section{Development duration from egg hatch to pupation}

The development duration from egg hatch to pupation is calculated as the days from when eggs are observed to hatch to the date when the first pupae are observed, or some variant, for example, to the median day of pupation. This is a compound measure indicating environmental conditions, fitness, and rearing density. The rate of development is determined by physical conditions, feeding, competition, and general fitness of the larvae. Development time is important in planning timing sequences and labor needs over several generations. A trend of increasing duration for larval development may mean that growing conditions are not good for the colony, for example, temperature is too low or too high, tray density is not consistent with protocols, or feeding is not consistent with protocols. Larval death or miscounting larvae could affect the larval density in trays. A decreasing trend in the larval development duration may mean that there is selection pressure for early development in the colony. Substantial variation in development duration could mean that there is inconsistency in temperature or feeding conditions.

\section{Pupal sex and trait ratio}

The pupal sexing process separates sexes into transgenic and nontransgenic groups reared from the larvae after the larval screen. This gives a number for males that are positive and negative for the fluorescent trait, and for females that are positive and negative for the fluorescent trait. From this, a sex ratio at the pupal stage is calculated. This sexing process verifies the proportion (e.g., 25\% expected) among the four 
groups (between the sexes in each fluorescence positive/ negative group). Deviation from the expected proportion in each of the four groups could mean there is a mistake in the sexing process, such as from a fault in fluorescent scanning equipment, or there has been sex-biased selection. If there is a consistent or substantial deviation in the sex ratio, possible causes of sex-biased survival in the rearing process may have occurred.

\section{Pupal eclosion rate}

The eclosion rate for adults is the proportion of pupae that survive to the adult stage. Eclosion rates are calculated from the number of live pupae initially collected from rearing trays, or a sample thereof, and the number of dead pupae remaining after three days. This indicator can be used to monitor any change in the survival of pupae. Trends of decreasing survival indicate poor growth conditions in the colonies, such as temperature outside the range specified in the protocol or an infection in the colony. Trends of increasing survival could indicate selection pressures that may mean groups of mosquitoes are inconsistent for use in laboratory studies over time.

\section{Wing length}

Wing length is a measure of distance between specific reference points on a mosquito wing. Wing length has been reported as a suitable proxy for overall body size and can be reliably measured directly or in photographs. This is a commonly used indicator of adult size (Lehman et al. 2006, Valerio et al. 2016), which is a compound measure of rearing conditions through all growth stages. Larger size is generally an indication of better growing conditions, and possibly greater fitness (for instance, due to more effectively competing for food), but it may also represent a potential incompatibility with normal wild populations with different size. Smaller size is generally an indication of poor growing conditions. Consistency in size is desirable over time. Wings are clipped, mounted on microscope slides and the distance between specific reference points on the wings is measured. Changes in wing length may be small over a generation, so this indicator may be measured at less frequent intervals (e.g., 4-6 generations). An increasing trend in size is most likely a result of overfeeding, or low larval density relative to food supply, and conversely for decreasing wing length. Food supply and larval density should be checked according to the mosquito rearing protocols.

\section{Insecticide resistance}

This is a measure of the mortality rate for standard WHO (2016) bioassays for a range of chemical insecticides used against adult and larval mosquitoes for which resistance is a documented concern in the countries where mosquitoes are held in containment. The insecticides of concern may vary, depending on reports of resistance (Knox et al. 2014). Insecticide resistance is an increasing problem in wild mosquito populations in Africa (Ransom and Lissenden 2016). A common concern about transgenic insects released into the environment is that they may have greater resistance to insecticides than wild populations. Laboratory rearing conditions would generally be expected to have no selective pressure specific to insecticide resistance, and, therefore, reared mosquitoes, either transgenic or wild type, would be expected to become less resistant than wild populations. The insecticide resistance status of both transgenic and wild-type colonies should be determined soon after they are established, annually thereafter, and immediately before any approved releases into the environment.

\section{Temperature, humidity, and lighting}

To interpret fluctuations in the quality indicators cited earlier, temperature, humidity and light regimes are controlled and recorded in the area of the laboratories where mosquitoes are reared. Lighting should be set to provide a day:night cycle and dusk/dawn transitions may also be relevant. Control settings should be checked to ensure lighting operates as intended. Temperature is a key driver of development and survival for all mosquito life stages in the laboratories, and humidity is important for adult survival. Temperature and humidity in the insectary rooms can be monitored continuously with automatic data loggers, set to record values at regular intervals (e.g., hourly). Where data loggers are not available, manual checks should be made regularly during the day and readings should be recorded. These should be recorded regularly to document conditions in the laboratory over time. Temperature and humidity must be maintained within specified ranges, following the mosquito rearing protocols. Values should be checked by the laboratory manager daily, and conditions should be adjusted if necessary to ensure they do not fall outside of intended ranges. Automatic monitoring devices can be set to send warnings when upper or lower thresholds are exceeded.

\section{Conclusion}

Transgenic mosquito colonies are held for many generations during the contained use stage of small-scale lab studies and scaling of production for regulated field releases. During this period, specific traits are checked for performance to ensure the experimental utility of the colony. A set of relevant quality indicators has been demonstrated to show quality over time, between labs and between strains. These quality indicators also enable laboratory managers to demonstrate conformity with specified characteristics in the colonies, which may support compliance, and to ensure risk assessments are based on reliable assumptions about the expected performance of mosquitoes contained during production.

\section{Acknowledgments}

The collaboration and discussions contributing to this review took place in 2016 and were funded by a grant from the Target Malaria project. Target Malaria receives core funding from the Bill \& Melinda Gates Foundation (BMGF) and from the Open Philanthropy Project Fund, an advised fund of Silicon Valley Community Foundation. The authors also wish to thank Jana Sikorova for document support.

\section{Author Disclosure Statement}

No competing financial interests exist. 


\section{References}

Alphey LS, Benedict MQ, Bellini R, Clark GG, et al. Sterileinsect methods for control of mosquito-borne diseases: An analysis. Vector Borne Zoonotic Dis 2010; 10:295-311.

Benedict MQ, Knols BGJ, Bossin HC, Howell PI, et al. Colonisation and mass rearing: Learning from others. Malar $\mathrm{J}$ 2009; 8 Suppl 2:S4.

Black WC, Alphey LS, James AA. Why RIDL is not SIT. Trends Parasitol 2011; 27:362-370.

Burt A. Heritable strategies for controlling insect vectors of disease. Philos Trans R Soc Lond B Biol Sci 2014; 369:20130432.

Charlwood JD. May the force be with you: Measuring mosquito fitness in the field. In: Takken W, Scott TW, eds. Ecological Aspects for Application of Genetically Modified Mosquitoes. Wageningen, The Netherlands: Kluwer, 2003:47-62.

Charlwood JD, Jones MDR. Mating in the mosquito, Anopheles gambiae sl. Physiol Entomol 1980; 5:315-320.

Crisanti A. INFRAVEC: Research capacity for the implementation of genetic control of mosquitoes. Pathog Glob Health 2013; 107: 458-462.

Davidson G, Odetoyinbo JA, Colussa B, Coz J. A field attempt to assess the mating competitiveness of sterile males produced by crossing 2 members of the Anopheles gambiae complex. Bull World Health Organ 1970; 42:55-67.

European Food Safety Authority. Guidance on the environmental risk assessment of genetically modified animals. EFSA J 2013; 11:3200

Facchinelli L, Valerio L, Lees RS, Oliva CF, et al. Stimulating Anopheles gambiae swarms: Application for behavioural and fitness studies. Malar J 2015; 14:271.

Facchinelli L, Valerio L, Ramsey JM, Gould F, et al. Field cage studies and progressive evaluation of genetically-engineered mosquitoes. PLoS Negl Trop Dis 2013; 7:e2001.

FAO/IAEA/USDA (Food and Agriculture Organization of the United Nations/International Atomic Energy Agency/United States Department of Agriculture). Manual for product quality control and shipping procedures for sterile mass-reared tephritid fruit flies, Version 5.0. 2003. Available at www.naweb.iaea.org/ nafa/ipc/public/QualityControl.pdf

Fried M. Determination of sterile-insect competitiveness. J Econ Entomol 1971; 64:869-872.

Galizi R, Doyle LA, Menichelli M, Bernardini F, et al. A synthetic sex ratio distortion system for the control of the human malaria mosquito. Nat Commun 2014; 5:3977.

Harvey-Samuel T, Ant T, Gong H, Morrison NI, et al. Population-level effects of fitness costs associated with repressible female-lethal transgene insertions in two pest insects. Evol Appl 2014; 7:597-606.

Irvin N, Hoddle MS, O'Brochta DA, Carey B, et al. Assessing fitness costs for transgenic Aedes aegypti expressing the GFP marker and transposase genes. Proc Natl Acad Sci U S A 2004; 101:891-896.

Isaacs AT, Jasinskiene N, Tretiakov M, Thiery I, et al. Transgenic Anopheles stephensi coexpressing single-chain antibodies resist Plasmodium falciparum development. Proc Natl Acad Sci U S A 2012; 109:e1922-e1930.

Knox TB, Juma EO, Ochomo EO, Jamet HP, et al. An online tool for mapping insecticide resistance in major Anopheles vectors of human malaria parasites and review of resistance status for the Afrotropical region. Parasit Vectors 2014; 7:76.

Lehmann T, Dalton R, Kim EH, Dahl E, et al. Genetic contribution to variation in larval development time, adult size, and longevity of starved adults of Anopheles gambiae. Infect Genet Evol 2006; 6:410-416.
Marchand RP. A new cage for observing mating behavior of wild Anopheles gambiae in the laboratory. J Am Mosq Control Assoc 1985; 1:234-236.

Okamoto KW, Robert MA, Gould F, Lloyd AL. Feasible introgression of an anti-pathogen transgene into an urban mosquito population without using gene-drive. PLoS Negl Trop Dis 2014; 8:e2827.

Oliva CF, Benedict MQ, Lempérière G, Gilles J. Laboratory selection for an accelerated mosquito sexual development rate. Malar J 2011; 10:135.

Paton D, Touré M, Sacko A, Coulibaly MB, et al. Genetic and environmental factors associated with laboratory rearing affect survival and assortative mating but not overall mating success in Anopheles gambiae sensu stricto. PLoS One 2013; 8:e82631.

Powell JR, Tabachnick WJ. Genetic shifting: A novel approach for controlling vector-borne diseases. Trends Parasitol 2014; 30:282-288.

Quinlan MM. Delivery of a novel intervention for vector control: Learning frameworks to support complex decisions. Thesis for PhD. Imperial College London, 2018.

Quinlan MM, Birungi J, Coulibaly M, Diabaté A, et al. Containment studies of transgenic mosquitoes in disease endemic countries: The broad concept of facilities readiness. Vector Borne Zoonotic Dis 2018; 18:14-20.

Ransom H, Lissenden N. Insecticide resistance in African Anopheles mosquitoes: A worsening situation that needs urgent action to maintain malaria control. Trends Parasitol 2016; 32: 187-196.

Reisen WK, Knop NF, Peloquin JJ. Swarming and mating behavior of laboratory and field strains of Culex tarsalis (Diptera: Culicidae). Ann Entomol Soc Am 1985; 78:667-670.

Scolari F, Siciliano P, Gabrieli P, Gomulski LM, et al. Safe and fit genetically modified insects for pest control: From lab to field applications. Genetica 2011; 139:41-52.

Valerio L, Collins CM, Lees RS, Benedict MQ. Benchmarking vector arthropod culture: An example using the African malaria mosquito, Anopheles gambiae (Diptera: Culicidae). Malar J 2016; 15:262.

WHO (World Health Organization). Test procedures for insecticide resistance monitoring in malaria vector mosquitoes. 2016. Available at http://apps.who.int/iris/bitstream/10665/ 250677/1/9789241511575-eng.pdf

WHO/TDR and FNIH (World Health Organization/Special Programme for Research and Training in Tropical Diseases and Foundation for the National Institutes of Health). Guidance framework for testing genetically modified mosquitoes. 2014. Available at www.who.int/tdr/publications/year/2014/ guide-fmrk-gm-mosquit/en

Yamada H, Vreysen MJ, Gilles JR, Munhenga G, et al. The effects of genetic manipulation, dieldrin treatment and irradiation on the mating competitiveness of male Anopheles arabiensis in field cages. Malar J 2014; 13:318.

Address correspondence to: John D. Mumford Imperial College London 1.08 Hamilton Building Silwood Park Campus Ascot, SL5 7PY Berkshire United Kingdom

E-mail: j.mumford@imperial.ac.uk 